ARTIGOS / ARTICLES

\title{
ANÁLISE DA CURVA DE PROGRESSO TEMPORAL DA VASSOURA-DE-BRUXA DO CUPUAÇUZEIRO*
}

\author{
ANGELA M. L. NUNES ${ }^{1}$, ARMANDO BERGAMIN FILHO'2, LILIAM AMORIM ${ }^{2}$, \\ MARCO AURÉLIO L. NUNES ${ }^{3} \&$ CARLOS T. S. DIAS ${ }^{4}$
}

\begin{abstract}
'Embrapa Rondônia, Cx. Postal 406, CEP 78.900-970, Porto Velho, RO, e-mail: angela@cpafro.embrapa.br; ${ }^{2}$ Departamento de Entomologia, Fitopatologia e Zoologia Agrícola; ${ }^{3}$ Departamento BVF, Faculdade de Ciências Agrárias do Pará, Cx. Postal 917, CEP 66.077-530, Belém, PA; ${ }^{4}$ Departamento de Ciências Exatas, Escola Superior de Agricultura "Luiz de Queiroz" Universidade de São Paulo, Cx. Postal 9, CEP 13.418-900, Piracicaba, SP
\end{abstract}

(Aceito para publicação em 20/11/2001)

Autor para correspondência: Angela Maria Leite Nunes

NUNES, A.M.L., BERGAMIN FILHO, A., AMORIM, L., NUNES, M.A.L. \& DIAS, C.T.S. Análise da curva de progresso temporal da vassoura-de-bruxa do cupuaçuzeiro. Fitopatologia Brasileira 27:344-348. 2002.

\section{RESUMO}

A evolução de lançamentos e de flores de cupuaçuzeiro (Theobroma grandiflorum) com sintomas de vassoura-de-bruxa e a produção de basidiocarpos do patógeno (Crinipellis perniciosa) foram monitoradas em plantas enxertada e não-enxertadas de um campo experimental no município de Belém, PA. Modelos epidemiológicos (logístico, Gompertz, e as somas logístico + logístico e Gompertz + Gompertz) foram ajustados a curvas de progresso da doença bianuais (1993 e 1994 para incidência da doença em lançamentos e basidiocarpos produzidos nesses lançamentos) e anuais (1994), para incidência da doença em flores e basidiocarpos produzidos nessas flores. A soma de duas equações logísticas apresentou bom ajuste aos dados bianuais e o modelo logístico, aos dados anuais. O início da manifestação dos sintomas esteve, geralmente, associado ao período seco enquanto que a produção de basidiocarpos coincidiu com o período chuvoso. A maior emissão de vassouras-de-bruxa ocorreu de julho a outubro e a maior frutificação, entre maio e julho.

Palavras-chave adicionais: Theobroma grandiflorum, Crinipellis perniciosa, modelos de progresso de doenças.

ABSTRACT

\section{Analyses of the temporal progress curve of "cupuaçuzeiro" witches' broom}

An experimental field study was carried out in Belém, Pará, Brazil, in order to monitor the evolution of two diseases in grafted and non-grafted plants: 1) the amount of cupuaçuzeiro (Theobroma grandiflorum) sprouts and flowers with symptoms of witches' broom and 2) the presence of basidiocarps of the pathogen Crinipellis perniciosa. Epidemiological models (logistic, Gompertz, logistic + logistic and Gompertz + Gompertz) were fit to biannual disease progress curves (1993 and 1994 for the incidence of the disease in the sprouts and for basidiocarp production in the sprouts) and annual (1994) for the disease incidence on flowers and for basidiocarps produced in the flowers. The sum of the two logistic equations fit well with biannual data and the logistic model fit the annual data. The appearance of symptoms was generally associated with the dry period, whereas the production of basidiocarps occurred during the rainy period. The amount of witches' broom was highest from July to October and the most fungus fruiting bodies was detected in May-July.
A vassoura-de-bruxa, causada por Crinipellis perniciosa (Stahel) Singer, é a principal doença do cupuaçuzeiro [Theobroma grandiflorum (Willd. ex. Spreng) Schum]. O ataque do patógeno nos ramos do ano leva à hiperplasia e hipertrofia, tendo como conseqüência perda na produção visto que esta frutífera produz no ramo do ano.

Os sintomas são semelhantes aos descritos na cultura do cacau (Andebrhan et al., 1983). Nos brotos vegetativos infetados o patógeno acompanha o ponto de crescimento,

\footnotetext{
*Parte da Tese de Doutorado do primeiro autor. Escola Superior de Agricultura "Luiz de Queiroz" (2000)
}

tornando-os hipertrofiados e com intensa proliferação lateral, cujo aspecto característico é de uma vassoura-de-bruxa. Após, determinado período, as vassouras-de-bruxa secam, as folhas enrolando-se, ficam dependuradas, destacando-se entre as ramagens e a coloração verde da planta. Quando a infecção ocorre nos primeiros estádios de formação do broto floral, há formação de ramalhete de flores anormais, hipertrofiadas e agigantadas. As flores hipertrofiadas dão origem a frutos deformados que morrem prematuramente. Nos frutos, os sintomas variam dependendo do modo de infecção e da idade do mesmo. Destacam-se dois tipos de infecção: a) indireta, através das flores infetadas dando origem a frutos de formato 
Análise da curva de progresso temporal da vassoura-de-bruxa...

cilíndrico com o centro deprimido de coloração pardo-escura e petrificado; b) direta, por meio do epicarpo. Frutos infetados ainda jovens adquirem a forma elipsóide. Frutos infetados em estádio mais desenvolvido apresentam, quando adultos, manchas de coloração parda. Nesse caso, as amêndoas encontram-se parcialmente ou totalmente apodrecidas e aderidas entre si.

Segundo Fry (1982), o conhecimento do desenvolvimento de uma doença em populações é importante para auxiliar na escolha de estratégia de controle e para avaliar o efeito das estratégias adotadas. O progresso temporal de doenças policíclicas tem sido descrito por modelos sigmóides simples, como os logísticos e de Gompertz, por exemplo, e por modelos sigmóides duplos, obtidos pela soma desses modelos simples (Hau et al. 1993; Bergamin Filho \& Amorim, 1996). Segundo Hau et al. (1993) vários patossistemas apresentam padrão de crescimento sigmóide duplo, como Ustilago scitaminea Sydow-cana-de-açúcar (Saccharum sp.) (Amorim \& Bergamin Filho, 1991; Amorim et al., 1993), Phytophthora capsici Leonian-pimentão (Capsicum annuum L.) (Bowers et al., 1990) e Fusarium oxysporum f. sp. vasinfectum (Atk.) Snyder \& Hansen combinado com Meloidoyine incognita (Kofoid \& White) Chitwood-algodão (Gossypium hirsutum L.) (Starr, 1989). Esses modelos de crescimento podem, ainda, ajustar-se às curvas poliéticas de progresso de doenças para clorose variegada dos citros ( Citrus spp.) (Laranjeira et al., 1998).

Considerando a escassez de informações sobre a dinâmica da vassoura-de-bruxa do cupuaçuzeiro, este trabalho, objetivou a avaliação temporal do progresso da vassoura-de-bruxa do cupuaçuzeiro, em órgãos vegetativos e reprodutivos.

\section{Descrição da área}

O progresso da vassoura-de-bruxa do cupuaçuzeiro quantificado por incidência (com as variáveis $n^{\circ}$ de vassourade-bruxa em lançamentos e em flores) e produção de basidiocarpos foi monitorado em plantas de cupuaçuzeiro em sistema sombreado, no campo experimental de fruteiras da Embrapa Amazônia Oriental, Belém-PA (latitude de $01^{\circ} 28^{\prime} \mathrm{O}$; longitude $48^{\circ} 28^{\prime} \mathrm{S}$ e a $10 \mathrm{~m}$ de altitude).

O campo experimental foi implantado em 1984, com material enxertado e não-enxertado. O enxertado foi proveniente da seleção da mesma planta mãe, mais produtiva; o não-enxertado foi obtido de processo de multiplicação de sementes, coletadas das plantas mais produtivas.

\section{Obtenção dos dados}

Os dados para os estudos nos órgãos vegetativos foram coletados em 20 plantas, dez enxertadas e dez não-enxertadas. Em cada planta foram marcados, nos três terços; superior, médio e inferior, no sentido horário, dez ramos, perfazendo um total de 200 ramos. Durante 1993 e 1994, os lançamentos com sintomas da doença foram contados e etiquetados semanalmente.

Para a coleta nos órgãos reprodutivos, em 1994, foram previamente selecionadas ao acaso quatro plantas nãoenxertadas na mesma área experimental. Em cada planta, semanalmente foram etiquetadas e numeradas todas as flores com sintoma de vassoura-de-bruxa.

Os dados de produção de basidiocarpos, foram coletados nas vassouras-de-bruxa necróticas, numeradas e etiquetadas nas plantas marcadas citadas acima. As coletas foram diárias, contando-se o número de basidiocarpos produzidos em vassouras-de-bruxa vegetativas e reprodutivas. Após a contagem, os basidiocarpos eram eliminados. Nas vassouras-de-bruxa vegetativas, a contagem do número de basidiocarpos foi em 1994 e 1995. Já nas vassouras-de-bruxa reprodutivas, a contagem do número de basidiocarpos ocorreu apenas em 1995.

\section{Modelos e análise temporal do progresso da vassoura-de- bruxa do cupuaçuzeiro}

De acordo com Hau et al. (1993) ao se assumir que as curvas de dupla sigmóide resultam de dois processos de crescimento consecutivos, então as curvas podem ser obtidas pela soma de duas funções de crescimento simples. Numa primeira fase, a análise temporal constou da plotagem dos dados, progresso da doença em função do tempo (semanas) para lançamentos, flores e basidiocarpos. Na fase seguinte, diferentes modelos epidemiólogicos foram ajustados para o progresso semanal da vassoura-de-bruxa (lançamentos, flores e basidiocarpos). Os modelos logístico $+\log$ sístico $\left(\mathrm{Y}_{\mathrm{LL}}=\mathrm{p}_{11}\right.$ I $\left.\left\{1+\exp \left[-\left(\mathrm{p}_{12}+\mathrm{p}_{13} * \mathrm{t}\right)\right]\right\}+\mathrm{p}_{21} /\left\{1+\exp \left[-\left(\mathrm{p}_{22}+\mathrm{p}_{23} * \mathrm{t}\right)\right]\right\}\right)$ e Gompertz $+\operatorname{Gompertz}\left(\mathrm{Y}_{\mathrm{GG}}=\mathrm{p}_{11} \exp \left\{-\exp \left[-\left(\mathrm{p}_{12}+\mathrm{p}_{13} * \mathrm{t}\right)\right]\right\}+\mathrm{p}_{21} \exp \{-\exp [-\right.$ $\left.\left.\left.\left(\mathrm{p}_{22}+\mathrm{p}_{23} * \mathrm{t}\right)\right]\right\}\right)$ foram testados para vassouras-de-bruxa (lançamentos) e basidiocarpos provenientes de vassouras-debruxa secas, vegetativas. Os parâmetros $\mathrm{p}_{11}$ e $\mathrm{p}_{21}$ são as maiores assíntotas, $p_{12}$ e $p_{22}$, a incidência inicial da doença e $p_{13}$ e $p_{23}$ são as taxas de progresso dos dois processos e $\mathrm{t}=$ tempo (Hau et al., 1993).

Para flores com sintoma da doença e basidiocarpos produzidos nessas flores foram testados os modelos logístico $\left(\mathrm{Y}_{\mathrm{L}}=\mathrm{p}_{11} /\left\{1+\exp \left[-\left(\mathrm{p}_{12}+\mathrm{p}_{13} * \mathrm{t}\right)\right]\right\}\right)$ e $\operatorname{Gompertz}\left(\mathrm{Y}_{\mathrm{G}}=\mathrm{p}_{11} \exp \{-\right.$ $\left.\left.\exp \left[-\left(\mathrm{p}_{12}+\mathrm{p}_{13} * \mathrm{t}\right)\right]\right\}\right)$, onde $\mathrm{p}_{11}$ é a maior assíntota, $\mathrm{p}_{12}$ a incidência inicial da doença e $\mathrm{p}_{13}$ a taxa do processo e $\mathrm{t}=$ tempo (Campbell \& Madden, 1990). O modelo monomolecular não foi testado, pois a simples observação visual dos dados foi suficiente para descartá-lo.

O grau de ajuste de cada modelo foi avaliado em função dos valores do coeficiente de determinação $\left(R^{2}\right)$, soma de quadrado do resíduo (SQR) e estimativa da maior assíntota (AS) (Hau et al., 1993; Bergamin Filho \& Amorim, 1996). A regressão não-linear foi feita por meio do método QuaseNewton, que estima assintoticamente. Os programas STATISTICA (Stat Soft, Tulsa - OK - U.S.A.) e PLOT IT (Scientific the Programming Enterprises Hasleft) foram usados na análise e construção dos gráficos, respectivamente.

\section{Curvas de progresso da doença}

Os valores não-cumulativos e cumulativos da incidência da vassoura-de-bruxa do cupuaçuzeiro (Figura 1) 
A.M.L. Nunes et al.
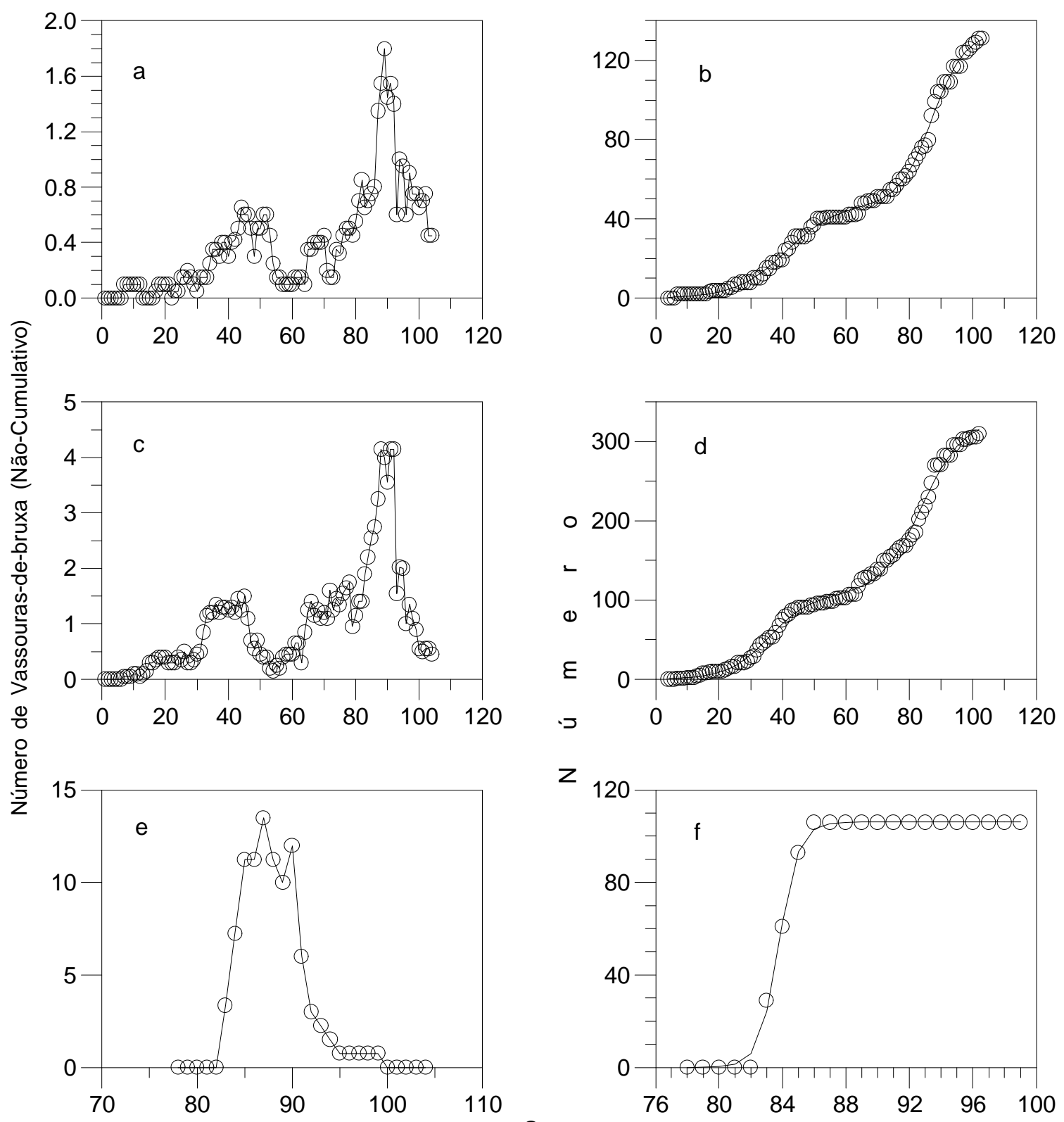

FIG. 1 - Curvas não-cumulativas, média mensal, (à esquerda), cumulativas e ajuste epidemiológico (à direita - linha contínua) do progresso da incidência da vassoura-de-bruxa. (a, b) plantas de cupuaçuzeiro (Theobroma grandiflorum) enxertadas; (c, d) plantas de cupuaçuzeiro nãoenxertadas e (e, f) flores. No período de 1993 a 1994.

evidenciaram maior incidência de lançamentos com sintomas da doença entre as semanas 30 e 40 e 80 e 100 tanto nas plantas enxertadas e quanto as não-enxertadas, que correspondem, respectivamente, aos meses de agosto a outubro e de julho a dezembro. Nas flores, a maior incidência da doença foi entre as semanas 80 e 100 (Figura 1). Já para basidiocarpos (Figura 2), o maior número ocorreu entre as semanas 70 e 80 e 120 e 132 tanto nas plantas enxertadas como nas não-enxertadas, que corresponde aos meses de maio a julho e abril a julho, respectivamente (Figura 2). Nas flores a maior produção de basidiocarpos ocorreu entre as semanas
120 e 132 (Figura 2). As curvas cumulativas exibem semelhança, entre plantas enxertadas e não-enxertadas (Figuras 1 e 2) diferindo unicamente no nível assintótico das duas fases de crescimento da doença.

O período latente, determinado pela diferença entre as curvas de sintomas iniciais e as curvas de produção de basidiocarpos, foi estimado entre 15 e 61 semanas. O período infeccioso, determinado pela diferença entre o início da produção de basidiocarpos e a deterioração da vassoura-debruxa, foi estimado entre duas e 48 semanas.

O início da epidemia da vassoura-de-bruxa do 
Análise da curva de progresso temporal da vassoura-de-bruxa...
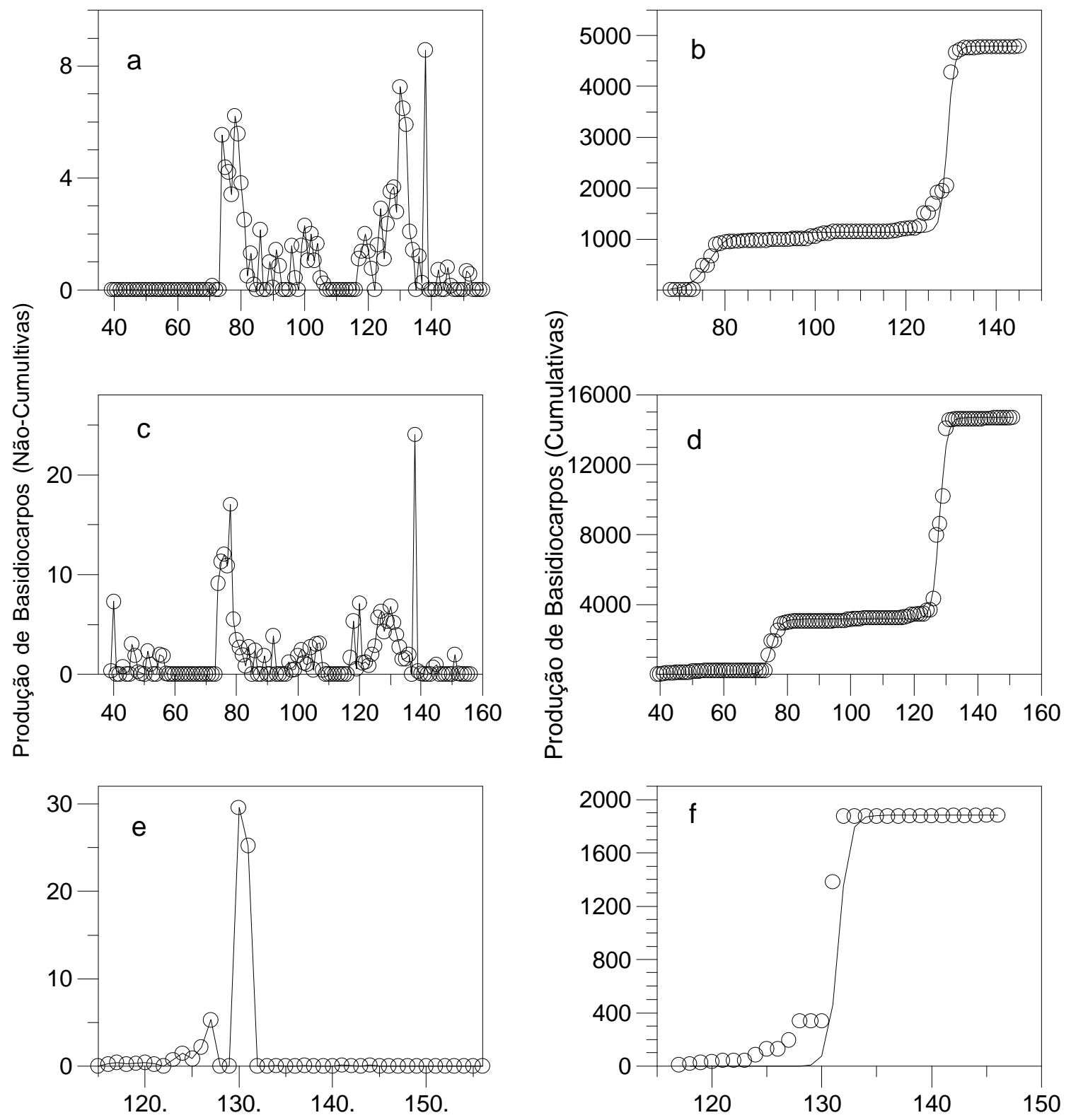

FIG. 2 - Curvas não-cumulativas, média mensal, (à esquerda); cumulativas e ajuste epidemiológico (à direita - linha contínua) do progresso da produção de basidiocarpos. (a, b) plantas de cupuaçuzeiro (Theobroma grandiflorum) enxertadas, $(c, d)$ plantas de cupuaçuzeiro nãoenxertadas e (e, f) flores. No período de outubro de 1994 à dezembro de 1995.

cupuaçuzeiro esteve geralmente associado ao período seco (lançamentos e flores) enquanto a produção de basidiocarpos iniciou-se com o período chuvoso. A maior emissão de vassouras-de-bruxa (lançamentos e flores) ocorreu de julho a outubro e a maior frutificação entre maio e julho. Na segunda fase do progresso maiores valores cumulativos semanais de lançamentos doentes e de basidiocarpos foram observados (Figuras 1 e 2). As plantas enxertadas apresentaram menor incidência da doença, tanto na primeira como na segunda fase do progresso. Isto deve-se, provavelmente, a um maior grau de resistência à doença nessas plantas, devido, provavelmente, a uma menor quantidade de doenças observado nas enxertadas.

De forma similar ao obtido por Evans \& bastos (1979) no patossistema Theobroma cacao L.-C. perniciosa, a maior incidência de vassoura-de-bruxa em lançamentos e flores coincidiu com o período de menor precipitação pluviométrica (agosto a outubro). A maior produção de basidiocarpos ocorreu entre maio e julho, coincidindo com o final do período chuvoso. A vassoura-de-bruxa do cupuaçuzeiro é altamente influenciada pelas condições ambientais, as quais foram representadas por análise de correlação canônica (Nunes, 
2000). Resultados semelhantes também foram descritos, na cultura do cacau (Andebhran, 1985; Aranzazu \& Buritica, 1993; Costa et al.,1997).

A aplicação das funções de sigmóide dupla e sigmóide simples é proposta neste trabalho para a descrição da curva de progresso da vassoura-de-bruxa e permite mostrar que as mais importantes fontes de inóculo de C. perniciosa, são as vassouras-de-bruxa necróticas, provenientes de lançamentos, pois o número de basidiocarpos produzido nos órgãos vegetativos chega a ser oito vezes maior que nos reprodutivos (Figura 2).

O método de controle mais comum, a poda fitossanitária, reduz o inóculo inicial. A remoção dos tecidos doentes deve ocorrer em setembro-outubro, coincidentemente com a maior taxa de progresso da doença, no período seco. É também recomendável que aliada à poda de limpeza das árvores realizada nos meses de fevereiro-março, outra poda de remoção de tecidos doentes seja feita, evitando, assim, a produção de basidiocarpos que se inicia nos meses seguintes.

\section{REFERÊNCIAS BIBLIOGRÁFICAS}

AMORIM, L. \& BERGAMIN FILHO, A. Sugarcane smut development models:I. Annual curves of disease progress. Zeitschrittfur Pflanzenkrankheitenschutz und Pflanzenschutz 98:605-612. 1991.

AMORIM, L., BERGAMIN FILHO, A. \& HAU, B. Analysis of progress curves of sugarcane smut on different cultivars using functions of double sigmoid pattern. Phytopathology 83:933936. 1993.

ANDEBRHAN, T. Produção de basidiocarpos em relação ao controle fitossanitários. In: Comissão Executiva do Plano da Lavoura Cacaueira /Departamento Especial da Amazônia. Informe de Pesquisa de 1985. pp. 42-44.

ANDEBRHAN, T., ALMEIDA, L.C. de \& FONSECA, S.E.A. Doenças do cacaueiro. Belém:CEPLAC/DEPEA/COPES.1983. ARANZAZU, H.F. \& BURITICÁ, C.P. Comparative epidemiology
study:Colombia, Caldas e Llanos Orientales In: Rudgard S.A., Maddson A.C. \& Andebrhan, T. (Eds) Disease management in cocoa:comparative epidemiology of witches' broom. London:Chapman \& Hall. 1993. pp. 57-71.

BERGAMIN FILHO, A. \& AMORIM, L. Doenças de plantas tropicais:Epidemiologia e controle econômico. São Paulo. Ceres. 1996.

BOWERS, J.H., SONODA, R.M. \& MITCHELL, D.J. Path coefficient analysis of the effect of rainfall variables on the epidemiology of phytophthora bligt of pepper caused by Phytophthora capsici. Phytopathology 80:1439-1446. 1990.

CAMPBELL, C.L. \& MADDEN, L. Introduction to plant disease epidemiology. New York. Wiley, 1990.

COSTA, J.C.B., MAFFIA, L.A., ANDEBRHAN, T. \& CARVALHO, A.L.P. Produção de basidiocarpos de Crinipellis perniciosa em diferentes fontes de inóculo na região Amazônica. Fitopatologia Brasileira 22:507-512. 1997.

EVANS, H.C. \& BASTOS, C.N. Epidemiologia da vassoura-debruxa do cacaueiro. In: Comissão Executiva do Plano da Lavoura Cacaueira/Departamento Especial da Amazônia. Informe Técnico de 1979. pp. 32-33.

FRY, W.E. Priciples of plant disease management. New York. Academic Press, 1982.

HAU, B., AMORIM, L. \& BERGAMIN FILHO, A. Mathematical functions to describe disease progress curves of double sigmoid pattern. Phytopathology 83:928-932. 1993.

LARANJEIRA, F.F., BERGAMIN FILHO, A. AMORIM, L. \& BERGER, R.D. Aspectos práticos da epidemiologia da clorode variegada dos citro. Laranja 19:79-90. 1998.

NUNES, A.M.L. Análise do progresso temporal e variáveis climáticas associadas à vassoura-de-bruxa (Crinipellis perniciosa) do cupuaçuzeiro (Theobroma grandiflorum) (Willd. Ex. Spreng) Schum)). (Tese de Doutorado). Piracicaba, Escola Superior de Agricultura "Luiz de Queiroz"USP. 2000.

STARR, J.L., JEGER, M.J., MARTYN, R.D. \& SCHILLING, K. Effects of Meloidogyne incognita and Fusarium oxysporum f.s. vasinfectum on plant mortality and yield of cotton. Phytopathology 79:640-646. 